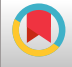

\title{
Reduction of Ovarian Radiation Dose and Image Quality Disruptive Effects: New Constructed Shields for CT Scanning of Abdomen and Pelvis
}

\author{
Mehran Bagheri ${ }^{1}$ and Mohammad Reza Azimi Aval ${ }^{1, *}$ \\ ${ }^{1}$ Department of Radiology, AJA Uiversity of Medical Sciences, Tehran, Iran \\ "Corresponding author: Department of Radiology, AJA University of Medical Sciences, Tehran, Iran. Email: dr.mr.azimi@gmail.com
}

Received 2020 August 31; Accepted 2020 September 29.

\begin{abstract}
Background: Cumulative dose of ovary as a radiosensitive organ during abdominal and pelvic CT scan imaging is still a controversial challenge that requires practical dose reduction strategies. Although bismuth shields can reduce the dose in the right proportions, their use is controversial due to the reduced image quality.

Objectives: The aim of this study was to investigate the performance of a new combination of X-ray absorber structures that have less effect on image quality parameters.

Methods: First, various shields with different weight percentages of $\mathrm{Cu}$ and Bi were made, then the percentage of dose reduction and image quality were evaluated via phantoms. Finally, Shield with the least effect on image quality was evaluated for clinical evaluation on 20 patients.

Results: Shielding with thicknesses of 1 T and 3T reduced the Entrance skin dose of ovarian by about 52\% and 73\%, respectively. Shields with $90 \%$ cu-10\% Bi and 100\% Bi structures had the least and most destructive effects on image quality, respectively, and also have the same image quality. The 10\% Bi-90\% Cu shield provided a $21 \%$ greater dose reduction than the bismuth shield. Also, this $1 \mathrm{~T}$ thick shield did not create an artifact in the reconstructed images.

Conclusions: Shields are flexible, inexpensive, and user-friendly for ovarian shielding in abdominal and pelvic CT scans. Unlike bismuth shields, shields do not have the detrimental effects of image quality degradation.
\end{abstract}

Keywords: MDCT, Ovary, Dose Reduction, Shielding

\section{Background}

The use of computed tomography (CT) as a diagnostic imaging technology has increased dramatically over the past two decades. CT scans have a higher radiation dose than other diagnostic methods, as this method is responsible for more than $70 \%$ of the total doses of x-ray diagnostic procedures $(1,2)$.

Multi-detector computed tomography(MCDT) is an advanced technology that is faster than older generations of CT scanners. However, more doses are given to the patient (3) due to the unique diagnostic role of CT scan in a huge range of abdominal and pelvic diseases. This method is considered to be the most accurate technique for abdominal and pelvic exams after ultrasound (4). We should bear in mind that the radiation dose on the patient's body surface is maximal, and it decreases as it passes through the patient's body (5). The dose of the ovary in a normal CT scan
Abdominal and pelvis were estimated in the range of 24.27 to 91.16 mGy via impact software with a standard deviation of $68.87 \pm 16.81(4)$.

Using a bismuth shield in CT imaging is an effective way to reduce the absorbed dose of ovaries $(6,7)$. Bismuth shield reduces the surface dose of the organ by absorbing low energy photons (8). Many studies have shown dose reduction efficiency of bismuth shields to be $26 \%$ to $57 \%$ (5, 6). In another study by Sancaktutar et al. (9), a dose reduction of approximately $90.2 \%$ was obtained using a bismuth shield for abdominal and pelvic CT scans. A dose of 50.5\% was obtained with bismuth shields (6).

On the other hand, despite the advantages of bismuth shields, some studies have shown the destructive properties of these shields on image quality parameters such as SNR and CT number, as well as creating artifacts (7, 9-12). 


\section{Objectives}

In this study, we designed and built shields that are perfectly suited for protecting the ovaries during CT scans of the abdomen and pelvis and do not have the disadvantages of common bismuth shield like reducing images quality and CT number shift that could disrupt the disease diagnose. Were clinically evaluated. Materials and methods, and then the results will be described below.

\section{Methods}

We used a tissue equivalent tissue phantom to measure the cumulative dose of ovarian position. This measurement was performed using thermoluminescence dosimeters. A CTDI acrylic phantom was used to measure noise and CT number changes. The effect of distance from the shield on image quality was investigated via the CTDI phantom to find the ideal distance. Image quality was also assessed using an anthropomorphic female phantom.

To evaluate the quality of the images, 20 female patients in the age range of $\mathbf{2 0}$ - 75 were divided into control and intervention groups. In the radiology department, after completing the consent forms, hardened shields were placed on the body of the ovary of women in the intervention group. A non-contrasting holder was made in which the shields were fixed, and under each shield was a $3 \mathrm{~cm}$ layer of noise-reducing foam between the patient's body and the shield. Routine abdominal and pelvic CT scans were performed in the control group of patients without any additional procedures.

All studies were performed using a Philips Brilliance 16slice CT scanner that was effective in axial position and 120 kvp tube voltage and 162 mAs tube current and slice thickness of $0.5 \mathrm{~cm}$

\subsection{Shield Construction, Image Quality Evaluation, and Statis- tical Analysis}

To reduce the patient dose without significantly reducing the SNR value, the photon energy must be intentionally modified so that low-energy beams can be removed from the spectrum, and high-energy beams can be maintained. To this end, physical protection may be a good solution. However, protective ingredients will play an important role in the success of this method. We found that $\mathrm{Cu}$ had all the properties and was selected as the base metal in the shield structure. Bismuth is a metal used as a shield in the past, while it cannot Modify the photon energy in a targeted way So that low energy photons remove from the spectrum and maintain high energy photons, which leads to image formation. Therefore, not a good choice. To test the accuracy and efficiency of this theory, different ovarian shields were made with different weight percentages of $\mathrm{Cu}$ and $\mathrm{Bi}$ (Table 1$)$.

\begin{tabular}{lccc}
\hline Table 1. Different Combinations and Widths of the Constructed Shields & \\
\hline Name & $\mathbf{B i}(\% \mathbf{W})$ & $\mathbf{C u}(\% \mathbf{W})$ & Width \\
\hline $\mathbf{1 0} \% \mathbf{B i - 9 0} \% \mathbf{C u}$ & 10 & 90 & $1 \mathrm{~T}-3 \mathrm{~T}$ \\
$\mathbf{5 0} \% \mathbf{B i - 9 0} \% \mathbf{C u}$ & 50 & 50 & $1 \mathrm{~T}-3 \mathrm{~T}$ \\
$\mathbf{9 0} \% \mathbf{B i}-\mathbf{- 1 0} \% \mathbf{C u}$ & 90 & 10 & $1 \mathrm{~T}-3 \mathrm{~T}$ \\
$\mathbf{1 0 0} \% \mathbf{B i}$ & 100 & 0 & $1 \mathrm{~T}-3 \mathrm{~T}$ \\
\hline
\end{tabular}

A mold made by 3D printing technology with a circular shape was used to shape the shields. RTV (roomtemperature-vulcanizing silicone) was mixed well as an elastomeric intermediate and $\mathrm{Cu}$ and Bi powders using a mechanical stirrer. The shields were made in a circular shape with a diameter of $120 \mathrm{~mm}$ and a constant thickness of $5 \mathrm{~mm}$ except at marginal $5 \mathrm{~mm}$.

The thickness edges decreases slightly to Avoid artifact due to the sharp edge of shields. For the preliminary evaluation of the new compounds, the effect of these shields on noise change and the CT number was investigated using CTDI phantom.

Quantitative image quality analyses performed via anthropomorphic phantoms by measuring noise and CT numbers shift at different ROIs. Different ROIs were evaluated. Image noise was measured using standard attenuation of attenuation values (per Hounsfield unit) in a homogeneous region. It was considered (Figure 1) that the CT number and standard deviation within each ROI were measured for ten consecutive sequences of exile images and then averaged to obtain an accurate value.

Differences between the groups were statistically determined to be less than 0.05 using paired student $t$-test and statistical significance. Kolmogorov-Smirnov test was performed to check the normality of the data. In the field of clinical trial, statistical differences between the two groups for image quality were assessed using the nonparametric Mann-Whitney test.

\section{Results}

\subsection{Surface Dose Using Anthropomorphic Phantom}

Table 2 shows the mean value and standard deviation of the skin input dose (ESD) measured for different shields 


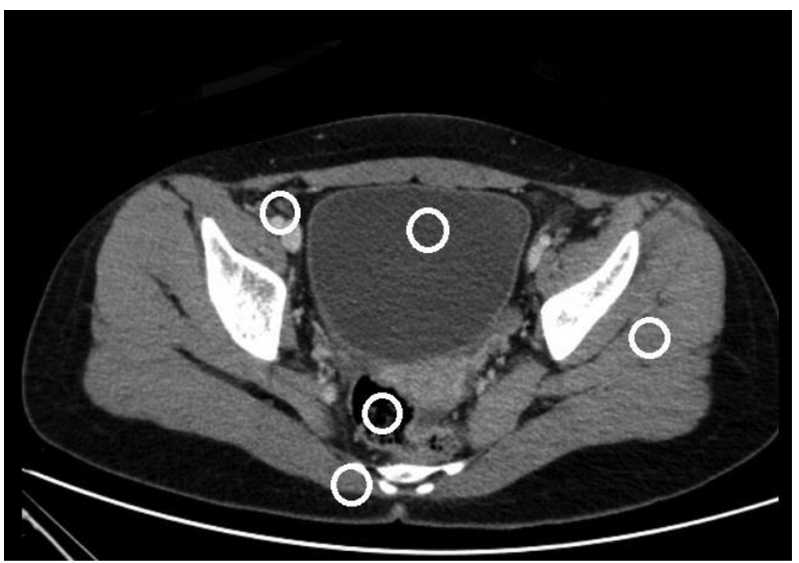

Figure 1. Selected different ROI, $s$ in pelvis axial CT scan for noise and CT number shift calculation

with thicknesses of 1T and 3T. The mean of ovarian ESD during abdominal and pelvic CT scan was $22.02 \pm 1.12$ for nonshielded condition and for 1T thickness, the amount of ESD reduction for $10 \% \mathrm{Bi}-90 \% \mathrm{Cu}, 50 \% \mathrm{Bi}-50 \% \mathrm{Cu}, 90 \% \mathrm{Bi}-10 \% \mathrm{Cu}$, and $100 \%$ Bi shields were shown to be $52.1 \%, 54.1 \%, 50.8 \%$, and $52.8 \%$, respectively.

\begin{tabular}{|c|c|c|c|}
\hline & Shield Composition & ESD, mGy & $\begin{array}{c}\text { Dose Re- } \\
\text { duction, } \\
\%\end{array}$ \\
\hline \multirow{5}{*}{$\mathbf{1 T}$} & No shielding & $22.2 \pm 1.12$ & - \\
\hline & $10 \% \mathrm{Bi}-90 \% \mathrm{Cu}$ & $10.55 \pm 0.91$ & 52.1 \\
\hline & $50 \% \mathrm{Bi}-50 \% \mathrm{Cu}$ & $10.06 \pm 0.77$ & 54.1 \\
\hline & $90 \% \mathrm{Bi}-10 \% \mathrm{Cu}$ & $10.82 \pm 0.74$ & 50.8 \\
\hline & $100 \% \mathrm{Bi}$ & $10.38 \pm 0.98$ & 52.8 \\
\hline \multirow{4}{*}{$3 T$} & $10 \% \mathrm{Bi}-90 \% \mathrm{Cu}$ & $5.8 \pm 1.1$ & 73.6 \\
\hline & $50 \% \mathrm{Bi}-50 \% \mathrm{Cu}$ & $5.85 \pm 0.63$ & 73.4 \\
\hline & $90 \% \mathrm{Bi}-10 \% \mathrm{Cu}$ & $5.92 \pm 0.87$ & 73.1 \\
\hline & $100 \% \mathrm{Bi}$ & $6.14 \pm 0.85$ & 72.1 \\
\hline
\end{tabular}

Also, for 3T thickness, ESD reduction rate for $10 \% \mathrm{Bi}-90 \%$ $\mathrm{Cu}, 50 \% \mathrm{Bi}-50 \% \mathrm{Cu}, 90 \% \mathrm{Bi}-10 \% \mathrm{Cu}$ and $100 \% \mathrm{Bi}$ shields equal to $73.6 \%, 73.4 \%, 73.1 \%$ and $72.1 \%$, respectively were shown.

For all manufactured shields, the dose reduction rate was statistically significant $(\mathrm{P}<0.05)$. By increasing the thickness of the shields from $1 \mathrm{~T}$ to $3 \mathrm{~T}$, a greater surface dose reduction rate (about $20 \%$ ) was obtained.
4.2. Image Quality Analysis Using CTDI Phantom and Anthropomorphic Phantom

The shields were placed on a uniform phantom to evaluate the quality of the image during the CT scan. Some ROIs were considered at the center of the phantom at different distances from the shield. The amount of CT number and noise were measured for each ROI in 10 consecutive slices, then the change in CT number and increase in noise were calculated comparing to the non-shielded mode (dose reference mode). Increasing the weight percentage of $\mathrm{Cu}$ in the structure of the shields reduces the noise and the amount of CT number shift in the images. 10\% Bi-90\% Cu Shield has the least effect on image quality in terms of noise and CT number shift, which decreases rapidly with increasing distance between shield and ROI.

\subsection{CT Number Shift and Noise Increasing}

The mean CT number and image noise on the anterior pelvic region were 11.9 and $6.8 \mathrm{HU}$, respectively. The use of 10\% Bi-90\% Cu, 50\% Bi-50\% Cu, 90\% Bi-10\% Cu, and 100\% Bi shields with 1T thickness, Anterior pelvic image noise 0.8 (11\%), 1.2, respectively (Increased by 17\%), 9.1 (27\%) and 7.2 (40\%). Mean CT of anterior pelvic CT for 1T thickness of 10\% Bi-90\% Cu, 50\% Bi-50\% Cu, 90\% Bi-10\% Cu, and 100\% Bi shields increased 5.8,14.4, 17.7, and 19.2 HU, respectively.

All composed shields except (10\% Bi-90\% Cu) "ideal shield" affected significantly in noise quantity and CT number shifting amount of all studied ROIs. The effects of the ideal shield with a thickness of $1 \mathrm{~T}$ had no statistically significant effect on bladder noise $(\mathrm{P}=0.08)$ and $\operatorname{rectal}(\mathrm{P}=0.07)$. Also, this shield did not significantly change the CT number of the gluteus maximus $(\mathrm{P}=0.53)$, iliac vessels $(0.06)$ and rectal $(\mathrm{P}=0.53)$. The effects of $3 \mathrm{~T}$ thickness ideal shield had no significant effect on rectal noise $(P=0.06)$ and iliac artery $C T$ number $(P=0.13)$. In addition, the effect of the ideal 3T thickness shield on image quality was similar to that of the 1T bismuth shield. Thus, the ideal 3T shield produced $21 \%$ more dose reduction properties than the $1 \mathrm{~T}$ bismuth shield in the same image quality.

The results showed that the ideal shield with a structure of $10 \% \mathrm{Bi}-90 \% \mathrm{Cu}$ shows the least effect on image quality for CT number shift and noise.

\section{Discussion}

The previous studies employed two techniques to calculate dose reduction: (ORGAN DOSE MEASURMENT or entrance skin dose (ESD) measurements), which in the present study, the second method was used. According to 
Dose measurements the use of shields made for ovaries with a thickness of 1T can significantly reduce the amount of ESD (50.8\% - 54.1\%). The dose reduction properties of 1T thick shields were approximately equal to the expected amount. The results of our dose reduction were consistent with the results of Hopper et al. (6), Alonso et al. (3), and Einstein et al. (11) studies, and the only difference with the mentioned studies could be related to imaging techniques. Performance of 1T constructed shields was better than study shields of Hohl et al. (13), Lambert and Gould (14); Yilmaz et al. (15), Gatuzzo (16), and Wang et al. (17).

Since the manufactured shields were effective, they can be used in clinical treatments. The efficiency of these shields increased significantly with increasing thickness from $1 \mathrm{~T}$ to $3 \mathrm{~T}$.

\subsection{Effect on Noise and CT Number Shift: An Anthropomorphic Phantom Study}

We used the CTDI phantom for the initial evaluation of the shields. The results showed that with increasing the percentage of $\mathrm{Cu}$, image noise and CT number shifting significantly decreased. Increasing the amount of $\mathrm{Cu}$ in shields from $0 \%$ to $90 \%$ leads to a $105 \%$ reduction in image noise dose and $21 \%$ in changing the CT number at a distance of $3 \mathrm{~cm}$ from the shield.

The results showed that the shield with a combination of $10 \% \mathrm{Bi}-90 \% \mathrm{Cu}$ will have the least detrimental effect on image quality. Both the made-up shield and the bismuth shield had a significant CT noise in direct contact with the phantom surface. However, as the distance between the shields and the phantom level increased, the noise and CT number increased rapidly. For the bismuth shield, the noise was reduced from $2296 \%$ to $85 \%$ by creating a distance of $5 \mathrm{~cm}$ relative to the direct contact with the phantom surface. Also, the CT number shifted from 161 to $33 \mathrm{HU}$.

By moving the ideal shields from contact to a distance of $5 \mathrm{~cm}$ from its surface via the phantom surface, the noise was reduced from $522 \%$ to $25 \%$, and the CT number change from 59 to $16 \mathrm{HU}$. These shields produced no noise and only resulted in a $6 \mathrm{HU}$ change in CT numbers at a distance of 9 $\mathrm{cm}$.

5.2. The Effect on Noise and CT Number: A "Phantom and Patient" Study

All shields were evaluated using a human-like phantom. The shields were placed on the ovarian region at a distance of $3 \mathrm{~cm}$. In the ROI of the gluteus intermedius region, the noise and CT number changes were $11 \%$ and 5.8 HU for the ideal shield, respectively, and $40 \%$ and $19.2 \mathrm{HU}$ for the bismuth shield, respectively. The ideal shield noise levels in the ROI of the bladder, rectum, and iliac arteries were $2 \%, 0.7 \%$, and $9 \%$, respectively. The ideal shield did not change the Cat number of the iliac artery and only resulted in a change of $2 \mathrm{HU}$ in the CT number in the bladder area and $1 \mathrm{HU}$ in the rectal area. The results of the study on the anthropomorphic phantom, like the results of the CTDI phantom study, proved that the ideal 1T-thick shield had no detrimental effect on image quality. Figure 2 shows a noise comparison diagram of the ideal shields and the bismuth shield at different ROIs.

The effects of $1 \mathrm{~T}$ thickness ideal shield on image quality were not significant in many areas. The ideal 3T thickness shield had the same effects as the 1T shield. However, the dose reduction in $3 \mathrm{~T}$ shields was $21 \%$ higher than in $1 \mathrm{~T}$ shield. Therefore, by using the ideal 3T shield instead of the conventional 1T bismuth shield, a dose reduction of $21 \%$ can be achieved with the same amount of image quality. The ideal 1T shield can also be used for better image quality. Figure 3 shows the effect of the two ideal and bismuth shields on the CT number of the ROI of the gluteus maximus and medius.

In the patient study, based on the radiologist report in Figure 4, the Ideal 1T shield did not have a detrimental effect on image quality, and the results of the clinical trial were consistent with studies on anthropomorphic phantoms.

\subsection{Patient Radiation Exposure Wasting}

As we know, radiation wasting, which means removing high energy photons from the radiation spectra, leads to a decrease in SNR, and a decrease in SNR has a linear relationship with an increase in noise. Therefore, a slight increase in image noise means that the amount of radiation wasting is not noticeable.

The results of this study showed that the amount of ideal shield noise is much less than the bismuth shield. Also, the amount of ideal shield noise in most ROI was not statistically significant (P value $<0.05$ ). It can be concluded that ideal shields have solved the problem of radiation loss.

\subsection{Conclusions}

The present study aimed to design and build a new shield to reduce the dose received by the ovaries in abdominal and pelvic CT scans without adversely affecting image quality. For this purpose, several shields were hardened using different combinations of $\mathrm{Cu}$ and $\mathrm{Bi}$, and their performance was compared with conventional bismuth shields. 


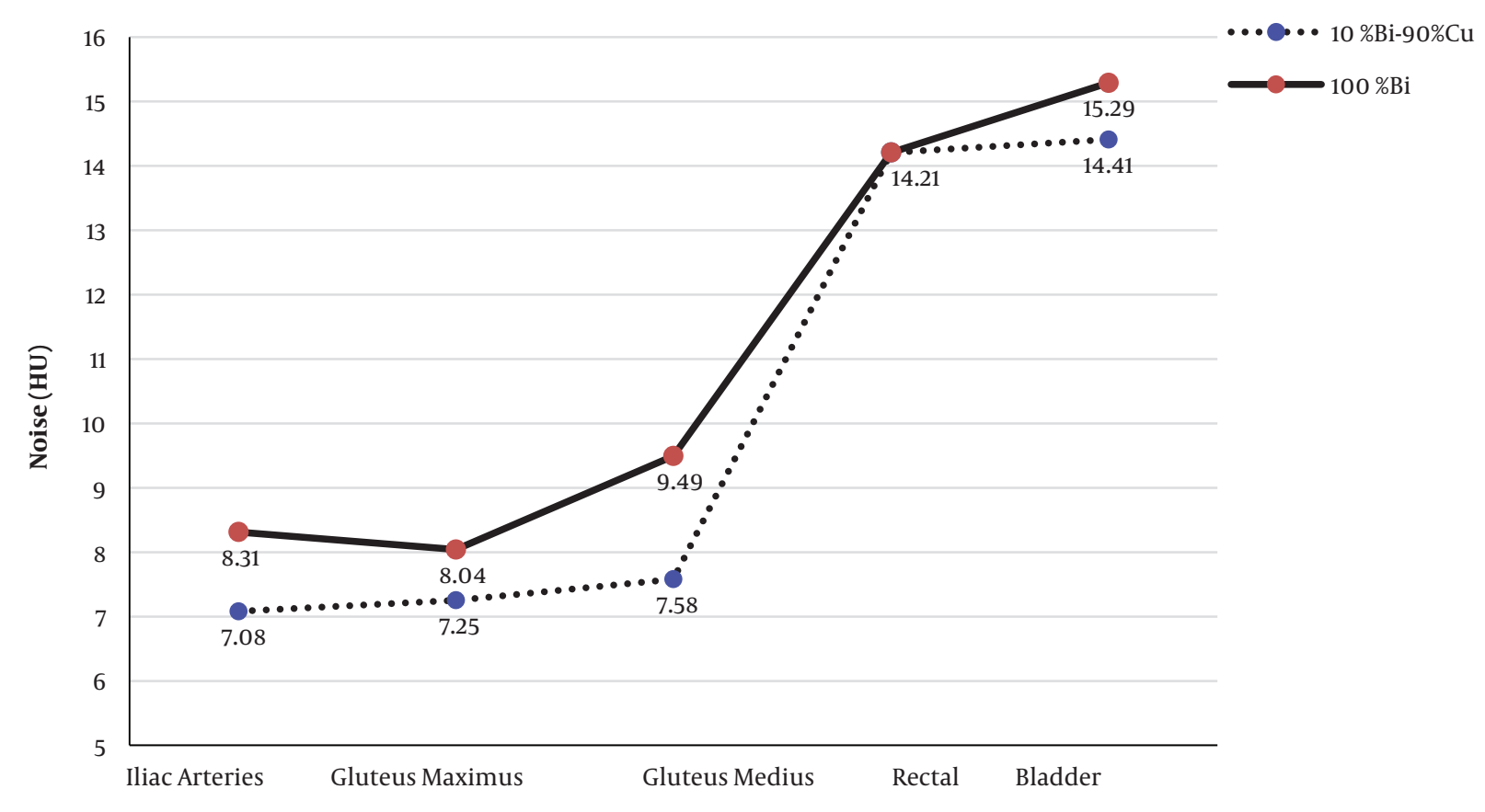

Figure 2. Comparison of 10\% Bi-90\% Cu and 100\% Bi shields on Images noise for different ROI

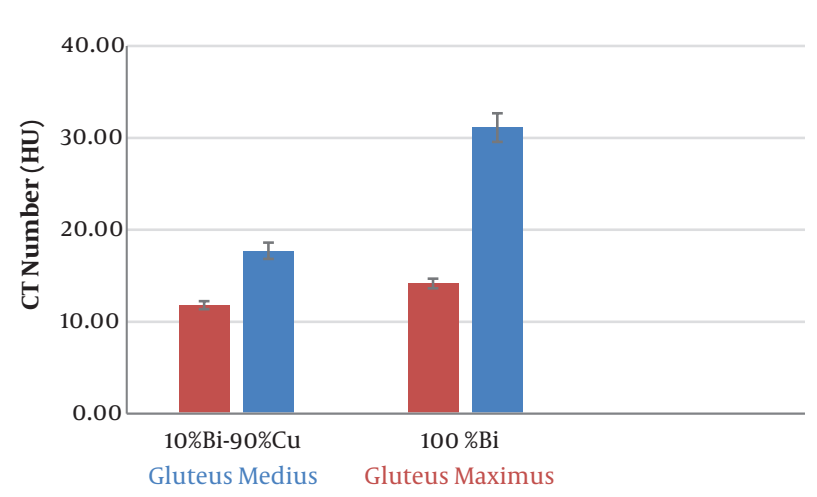

Figure 3. Comparison of 10\% Bi-90\% Cu and 100\% Bi on CT number shift for gluteu maximus and gluteus intermedius regions.

The results of studies on the uniform phantom of CTDI and anthropomorphic phantom showed that the shield with $10 \% \mathrm{Bi}-90 \% \mathrm{Cu}$ structure had the least effect on image quality. A new 1T-thick shield reduced the entry dose of ovarian skin by $50 \%$, similar to the bismuth 1T shield, while the effects of the ideal shield on image noise and CT change were calculated a lot less than the bismuth shield. Also, the ideal shield reduced the radiation dose by $21 \%$ more than the bismuth shield with the same thickness of $1 \mathrm{~T}$ in the same image quality.
In the anthropomorphic phantom study, the largest change in CT was observed for the ideal shield less than 6 HU.

Also, the maximum noise generated from the reference dose mode was less than $11 \%$. According to the radiologist, the results of the clinical trial were consistent with the results of phantom studies. On the other hand, no artifacts were observed in the reconstructed images for the Ideal $1 \mathrm{~T}$ Shield.

\section{Footnotes}

Authors' Contribution: Analysis and interpretation of data: Mehran Bagheri. Drafting of the manuscript: Mehran Bagheri critical revision of the manuscript for important intellectual content: Mohammad Reza Azimi Aval.

Conflict of Interests: All authors declare that they have no conflict of interest

\section{Ethical Approval: IR.AJAUMS.REC.1398.190}

Funding/Support: This study was supported in part by AJA University of Medical Sciences and by a teaching and from the Beesat hospital Physicians (Dr Mohammad Reza Azimi Aval). 

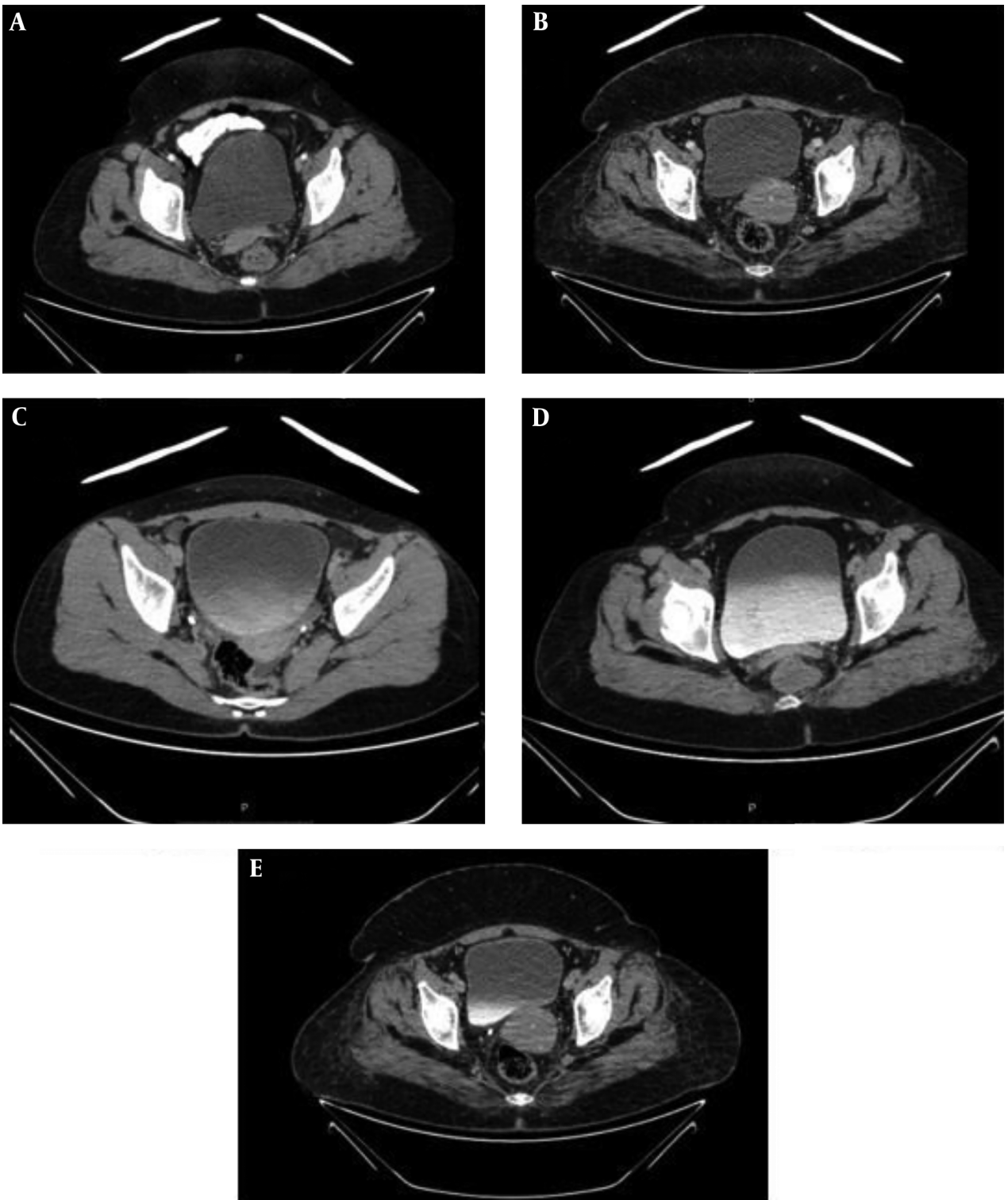

Figure 4. examples of pelvic CT images without and with 1T shields thicknesses. A, 100\% Bi; B, 90\% Bi-10\% Cu; C, 50\% Bi-50\% Cu; D, 10\% Bi-90\% Cu; E, without shielding.

\section{References}

1. Smith-Bindman R, Miglioretti DL, Johnson E, Lee C, Feigelson HS, Flynn M, et al. Use of diagnostic imaging studies and associated radiation exposure for patients enrolled in large integrated health care systems, 1996-2010. JAMA. 2012;307(22):2400-9. doi: 10.1001/jama.2012.5960. [PubMed: 22692172]. [PubMed Central: PMC3859870].

2. Pola A, Corbella D, Righini A, Torresin A, Colombo PE, Vismara L, et al. Computed tomography use in a large Italian region: trend analysis 2004-2014 of emergency and outpatient CT examinations in children and adults. Eur Radiol. 2018;28(6):2308-18. doi: 10.1007/s00330017-5225-x. [PubMed: 29318431].
3. Alonso TC, Mourao AP, Santana PC, da Silva TA. Assessment of breast absorbed doses during thoracic computed tomography scan to evaluate the effectiveness of bismuth shielding. Appl Radiat Isot. 2016;117:55-7. doi: 10.1016/j.apradiso.2016.03.018. [PubMed: 27033028].

4. Amirnia A, Mehnati P, Jabbari N. Estimation of absorbed dose of radiosensitive organs and effective sose in patients underwent $a b-$ dominopelvic spiral CT scan using impact CT patient dosimetry. Tehran Univ Med J. 2017;75(2):103-12.

5. Hopper KD, Neuman JD, King SH, Kunselman AR. Radioprotection to the eye during CT scanning. AJNR Am J Neuroradiol. 2001;22(6):1194-8. [PubMed: 11415918].

6. Hopper KD, King SH, Lobell ME, TenHave TR, Weaver JS. The breast: inplane x-ray protection during diagnostic thoracic CT-shielding with 
bismuth radioprotective garments. Radiology. 1997;205(3):853-8. doi: 10.1148/radiology.205.3.9393547. [PubMed: 9393547].

7. Lawrence S, Seeram E. The Current Use and Effectiveness of Bismuth Shielding in Computed Tomography: A Systematic Review. Radiol Open J. 2017;2(1):7-16. doi: 10.17140/roj-2-113.

8. Akhlaghi P, Miri-Hakimabad H, Rafat-Motavalli L. Effects of shielding the radiosensitive superficial organs of ORNL pediatric phantoms on dose reduction in computed tomography.J Med Phys. 2014;39(4):23846. doi: 10.4103/0971-6203.144490. [PubMed: 25525312]. [PubMed Central: PMC4258732].

9. Sancaktutar AA, Bozkurt Y, Onder H, Soylemez H, Atar M, Penbegul $\mathrm{N}$, et al. A new practical model of testes shield: the effectiveness during abdominopelvic computed tomography. J Androl. 2012;33(5):9849. doi: 10.2164/jandrol.111.015560. [PubMed: 22207708].

10. Mathieu KB, Cody DD. Partial arc beam filtration: a novel ap proach to reducing CT breast radiation dose. AJR Am J Roentgenol. 2013;200(3):601-7. doi: 10.2214/AJR.12.8771. [PubMed: 23436850]. [PubMed Central: PMC3855393].

11. Einstein AJ, Elliston CD, Groves DW, Cheng B, Wolff SD, Pearson GD, et al. Effect of bismuth breast shielding on radiation dose and image quality in coronary CT angiography. J Nucl Cardiol. 2012;19(1):100-8. doi: 10.1007/s12350-011-9473-x. [PubMed: 22068687]. [PubMed Central: PMC3266996].

12. Revel MP, Fitton I, Audureau E, Benzakoun J, Lederlin M, Chabi ML, et al. Breast dose reduction options during thoracic CT: influence of breast thickness. AJR Am J Roentgenol. 2015;204(4):W421-8. doi: 10.2214/AJR.14.13255. [PubMed: 25794091].

13. Hohl C, Wildberger JE, Suss C, Thomas C, Muhlenbruch G, Schmidt T, et al. Radiation dose reduction to breast and thyroid during MDCT: effectiveness of an in-plane bismuth shield. Acta Radiol.2006;47(6):5627. doi: 10.1080/02841850600702150. [PubMed:16875333].

14. Lambert JW, Gould RG. Evaluation of a Net Dose-Reducing OrganBased Tube Current Modulation Technique: Comparison With Standard Dose and Bismuth-Shielded Acquisitions. AJR Am J Roentgenol. 2016;206(6):1233-40. doi: 10.2214/AJR.15.15778. [PubMed: 27058461].

15. Yilmaz MH, Albayram S, Yasar D, Ozer H, Adaletli I, Selcuk D, et al. Female breast radiation exposure during thorax multidetector computed tomography and the effectiveness of bismuth breast shield to reduce breast radiation dose.JComput Assist Tomogr.2007;31(1):138-42. doi: 10.1097/01.rct.0000235070.50055.e6. [PubMed: 17259846].

16. Catuzzo P, Aimonetto S, Fanelli G, Marchisio P, Meloni T, Mistretta L, et al. Dose reduction in multislice CT by means of bismuth shields: results of in vivo measurements and computed evaluation. Radiol Med. 2010;115(1):152-69. doi: 10.1007/s11547-009-0469-4. [PubMed: 20012921].

17. Wang J, Duan X, Christner JA, Leng S, Yu L, McCollough CH. Radiation dose reduction to the breast in thoracic CT: comparison of bismuth shielding, organ-based tube current modulation, and use of a globally decreased tube current. Med Phys. 2011;38(11):6084-92. doi: 10.1118/1.3651489. [PubMed: 22047373]. 